Effects of thermal annealing on the structural, mechanical, and tribological properties of hard fluorinated carbon films deposited by plasma enhanced chemical vapor deposition

M. E. H. Maia da Costa, I. J. R. Baumvol, C. Radke, L. G. Jacobsohn, R. R. M. Zamora, and F. L. Freire

Citation: Journal of Vacuum Science \& Technology A 22, 2321 (2004); doi: 10.1116/1.1795833

View online: https://doi.org/10.1116/1.1795833

View Table of Contents: https://avs.scitation.org/toc/jva/22/6

Published by the American Vacuum Society

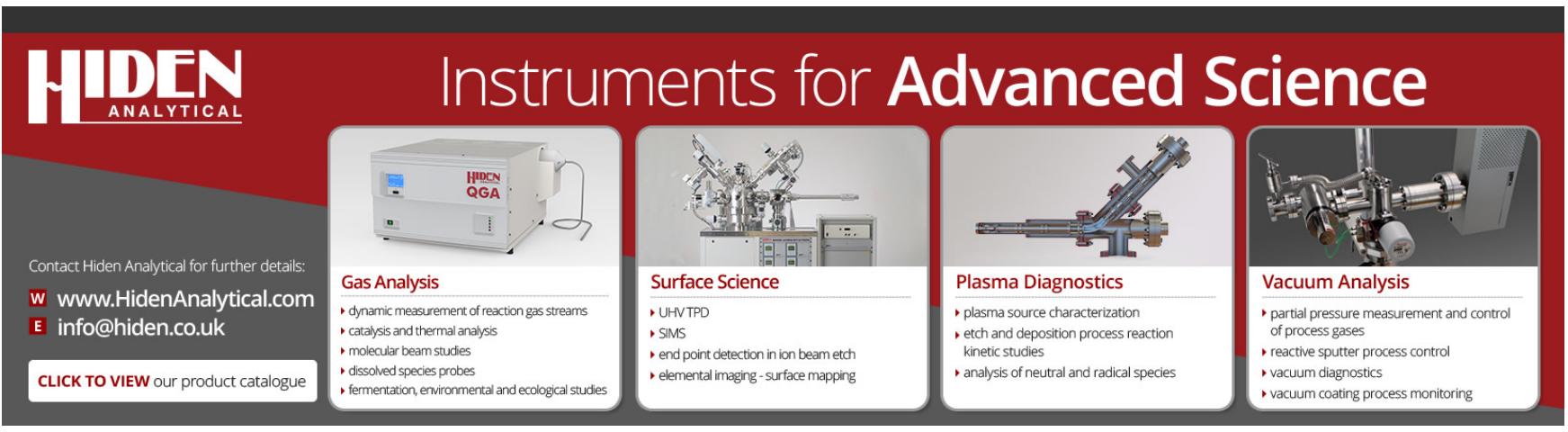




\title{
Effects of thermal annealing on the structural, mechanical, and tribological properties of hard fluorinated carbon films deposited by plasma enhanced chemical vapor deposition
}

\author{
M. E. H. Maia da Costa \\ Departamento de Física, Pontifícia Universidade Católica do Rio de Janeiro, Cx. Postal 3807, \\ Rio de Janeiro, RJ, 22453-970, Brazil \\ I. J. R. Baumvol and C. Radke \\ Instituto de Física, Universidade Federal do Rio Grande do Sul, Porto Alegre, RS, 91540-000, Brazil \\ L. G. Jacobsohn \\ Los Alamos National Laboratory, Materials Science and Technology Division, P. O. Box 1663, Los Alamos, \\ New Mexico 87545 \\ R. R. M. Zamora and F. L. Freire, Jr. ${ }^{\text {a) }}$ \\ Departamento de Física, Pontifícia Universidade Católica do Rio de Janeiro, Cx. Postal 3807, \\ Rio de Janeiro, RJ, 22453-970, Brazil
}

(Received 3 March 2004; accepted 26 July 2004; published 20 October 2004)

\begin{abstract}
Hard amorphous fluorinated carbon films (a-C:F) deposited by plasma enhanced chemical vapor deposition were annealed in vacuum for $30 \mathrm{~min}$ in the temperature range of $200-600{ }^{\circ} \mathrm{C}$. The structural and compositional modifications were followed by several analytical techniques: Rutherford backscattering spectrometry (RBS), elastic recoil detection analysis (ERDA), x-ray photoelectron spectroscopy (XPS) and Raman spectroscopy. Nanoidentation measurements and lateral force microscopy experiments were carried out in order to provide the film hardness and the friction coefficient, respectively. The internal stress and contact angle were also measured. RBS, ERDA, and XPS results indicate that both fluorine and hydrogen losses occur for annealing temperatures higher than $300{ }^{\circ} \mathrm{C}$. Raman spectroscopy shows a progressive graphitization upon annealing, while the surface became slightly more hydrophobic as revealed by the increase of the contact angle. Following the surface wettability reduction, a decrease of the friction coefficient was observed. These results highlight the influence of the capillary condensation on the nanoscale friction. The film hardness and the internal stress are constant up to $300{ }^{\circ} \mathrm{C}$ and decrease for higher annealing temperatures, showing a direct correlation with the atomic density of the films. Since the thickness variation is negligible, the mass loss upon thermal treatment results in amorphous structures with a lower degree of cross-linking, explaining the deterioration of the mechanical properties of the a-C:F films. () 2004 American Vacuum Society. [DOI: 10.1116/1.1795833]
\end{abstract}

\section{INTRODUCTION}

Hydrogenated amorphous carbon (a-C:H) is an interesting material due to its outstanding properties like high hardness, chemical inertness, high wear resistance and low friction. ${ }^{1} \mathrm{~A}$ way to further improve the properties of this material is by the incorporation of other elements during film growth. Among the many possibilities already investigated, the incorporation of fluorine has attracted the attention of the scientific community due to the possibility of lowering the internal stress, ${ }^{2}$ to suppress the $\mathrm{CH}_{n}$ absorption bands ${ }^{3}$ and to reduce the friction coefficient of a-C:H films. ${ }^{4,5}$ Therefore, fluorinated a-C:H (a-C:F) films present many possible applications such as wide-transparency window for optical devices operating in the visible and near-infrared part of the spectrum $^{3}$ and low friction and low surface energy coatings. ${ }^{4-11}$

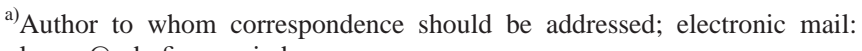
lazaro@vdg.fis.puc-rio.br
In spite of that, the motivation for the recent research on the fluorine incorporation into a-C:H films is the study of its electrical characteristics. In order to improve the switching performance of future ultra-large scale integrated circuits (ULSI), insulator films with dielectric constant lower than that of $\mathrm{SiO}_{2}$ are needed to reduce the capacitance of interlayer insulators. ${ }^{12}$ Fluorinated amorphous carbon films have been proposed as possible candidates due to their low dielectric constant. ${ }^{13-21}$ In fact, dielectric constants as low as 2.1 were achieved in plasma deposited film. ${ }^{12,13}$ In what concerns the application as interconnect dielectric material, the thermal stability is of major importance since it is submitted to thermal annealing during subsequent integrate circuit manufacturing steps. Among other requirements such as, gap-fill capability, adhesion and minimal moisture absorption, thermal stability up to $400{ }^{\circ} \mathrm{C}$ is necessary. ${ }^{12,18}$

The structure of a-C:F films has been investigated by several techniques which revealed that a progressive structural change from a diamond-like to a polymer-like material occurs when the concentration of fluorine-containing precursor 
gas in the deposition atmosphere is increased. ${ }^{22,23}$ Pulsed plasma deposition experiments reveal that by increasing the plasma excitation time the film character change from diamond-like to polymer-like. ${ }^{24}$ Moreover, it was observed that fluorine is incorporated into the films at the expenses of hydrogen. ${ }^{2}$ The skeleton of the films mostly consists of carbon rings connected by $\mathrm{CF}_{2}$ groups ${ }^{25}$ and films deposited at more intense ion bombardment assistance ${ }^{2}$ or high substrate temperatures show a high degree of interconnectivity. ${ }^{25}$

Motivated by the potential application of a-C:F films in ULSI devices, investigations of the thermal stability of a-C:F films were focused on the temperature range up to $400{ }^{\circ} \mathrm{C}$. With this purpose, dielectric constant, mass loss and thickness changing were monitored. However, despite some claims that plasma deposited carbon fluorinated films are thermally stable at those temperatures, the usefulness of a-C:F films have yet to be proved by integration in an ULSI structure. ${ }^{19}$ On the other side, experimental data concerning higher temperatures treatments are scarce. In particular, there are few data on the tribological and mechanical behavior upon annealing. In this way, Raman spectroscopy investigation has shown that progressive graphitization occurs at high annealing temperatures and that the transition temperature from the amorphous to a more graphitic-like structure decreases with the fluorine content of the films. ${ }^{26}$ Another study showed that an annealing-induced fluorine loss occurs by removing free radicals and unstable weak-bonded fragments trapped in the atomic network of the films. ${ }^{27}$ Strong effusion of $\mathrm{CF}_{4}$-related species is found to be consistent with a surface desorption process and can be observed when $\mathrm{CF}_{n}$ bonds are present in the film microstructure and the void network dimensions are large enough. ${ }^{28}$ In addition, infrared spectroscopy has revealed that $\mathrm{C}-\mathrm{F}_{3}$ and $\mathrm{C}-\mathrm{F}_{2}$ bonding configurations are weaker against thermal annealing than the $\mathrm{C}-\mathrm{F}$ configuration. ${ }^{16}$

In this work, we perform an investigation of the evolution of structural, mechanical and tribological properties of hard a-C:F film deposited by plasma enhanced chemical vapor deposition (PECVD) in an acetylene + carbon tetrafluoride gas mixture when submitted to vacuum thermal annealing with temperatures up to $600{ }^{\circ} \mathrm{C}$. Besides its importance for the understanding of the film microstructure, as well as a low-k dielectric material for ULSI devices applications, the study of thermal stability is crucial since it defines the temperature range in which the film can be used as protective coating. The deposition conditions were chosen in order to obtain hard fluorinated a-C:H films. ${ }^{29}$

\section{EXPERIMENTAL PROCEDURES}

Fluorinated carbon films were deposited by PECVD using a $\mathrm{C}_{2} \mathrm{H}_{2}-\mathrm{CF}_{4}$ (1:1) gas mixture in an asymmetrical capacitively coupled deposition system. The $p$-doped $\langle 100\rangle$-Si substrates were mounted on a water-cooled $7.5 \mathrm{~cm}$ diam copper cathode fed by a rf $(13.56 \mathrm{MHz})$ power supply. During the deposition, the temperature of the substrate holder was below $50{ }^{\circ} \mathrm{C}$, as measured by a thermocouple. The films were deposited with a total pressure of $10 \mathrm{~Pa}$ and a total influx of gas of $3 \mathrm{sccm}$. The self-bias voltage was $-350 \mathrm{~V}$ and the rf input power was $31 \mathrm{~W}$. In order to improve the adhesion of the film to the substrate, a thin $(10 \mathrm{~nm})$ buffer layer of a-C:H was deposited previously as described elsewhere. ${ }^{29}$ The typical film thickness was $250 \mathrm{~nm}$. The samples were annealed in a vacuum furnace with a base pressure lower than $10^{-4} \mathrm{~Pa}$. The annealing time was $30 \mathrm{~min}$, without any kind of sequential annealing on each sample. The temperature range was between 200 and $600{ }^{\circ} \mathrm{C}$.

The chemical composition was determined by ion beam analysis (IBA): Rutherford backscattering spectrometry (RBS) and elastic recoil detection analysis (ERDA) employing a 4 MV Van de Graaff accelerator KN-4000 from High Voltage Engineering Corp. For the RBS measurements, a $2 \mathrm{MeV} \mathrm{He}^{+}$beam was used with the particle detector positioned at $165^{\circ}$ with respect to the incident beam. ERDA measurements determined the hydrogen content using a $2.2 \mathrm{MeV}$ $\mathrm{He}^{+}$beam with the detector positioned at $30^{\circ}$, while the sample was tilted by $75^{\circ}$ with respect to the incident beam. The atomic density was determined by numerical simulation of the RBS spectra using the thickness and the hydrogen content determined by profilometry and ERDA, respectively, as input data.

X-ray photoelectron spectroscopy (XPS) was employed to investigate the chemical state of the carbon atoms at the films surface. It was performed using an Omicron UHV station based on an EA125 hemispherical analyzer. The photoelectron spectra of $\mathrm{C} 1 s$ and $\mathrm{F} 1 s$ core levels were monitored, excited by $\mathrm{Mg}-\mathrm{K} \alpha(1253.6 \mathrm{eV})$ radiation, with an overall resolution of $1 \mathrm{eV}$ for $10 \mathrm{eV}$ pass energy. The angle between surface normal and the electron energy analyzer axis was $45^{\circ}$. No surface cleaning procedure was carried out by means of argon sputtering in order to avoid hybridization changes of the carbon atoms or preferential sputtering due to $\mathrm{Ar}^{+}$bombardment.

The atomic arrangement of the films was probed by Raman scattering spectroscopy measurements that were performed with a Renishaw 2000 system using an $\mathrm{Ar}^{+}$-ion laser $(\lambda=514 \mathrm{~nm})$ in backscattering geometry. The laser power on the sample was $\sim 10 \mathrm{~mW}$ and the laser spot diameter was 2 to $3 \mu \mathrm{m}$. These measurements were carried out in air at room temperature.

Hydrophobicity was determined by contact angle measurements that were carried out with a Ramé-Hart goniometer and two different droplet sizes of Millipore purified and distilled water. The final values presented in this work correspond to the average of several independent measurements.

The surface root mean-square (rms) roughness was obtained by constant height contact atomic force microscopy (AFM) measurements employing a Nanoscope III equipment from Digital Instruments with a soft $(k=0.12 \mathrm{~N} / \mathrm{m})$ cantilever. Each data point corresponds to the average value of 8 measurements carried out in different $100 \times 100 \mathrm{~nm}^{2}$ spots with a contact force of about $80 \mathrm{nN}$. The friction measurements were carried out using the microscope in the lateral force regime and performed under controlled ambient conditions with room temperature and relative humidity of $23{ }^{\circ} \mathrm{C}$ 
and $38 \pm 1 \%$, respectively. The normal force acting at the tip-surface interface, which is composed of the adhesion force between the tip and sample plus the cantilever normal bending force, was kept constant by the microscope feedback system. The adhesion force was measured as the maximum negative bending force of the cantilever at a tip approach curve. The cantilever normal force was obtained by multiplying the measured cantilever bending by its normal bending constant. During the measurements, both images (lateral force forward and reverse) were acquired while the surface was scanned beneath the AFM $\mathrm{Si}_{3} \mathrm{~N}_{4}$ tip at an angle of $90^{\circ}$ from the cantilever main axis. In order to obtain the absolute values of the friction coefficients, the cantilever was calibrated using the Neumeister et al. formula, ${ }^{30}$ while the AFM was calibrated with the method proposed by Liu et al. ${ }^{31}$ In this way, the torsion of the AFM cantilever was correlated to the lateral deflection of the laser light on the photo detector by taken into account the optical path of the AFM laser light and geometrical optics. The normal and torsion bending constants of the cantilever were calculated as a function of its geometry and materials mechanical properties. The cantilevers dimensions were measured with the use of an optical microscope while the cantilever's thickness measured by scanning electron microscopy. A detailed comparison among these and other two calibration techniques can be found elsewhere. ${ }^{32}$ While numerous investigators have reported differences in the raw data gathered from day to day, a function perhaps of variations in the experimental conditions (humidity, tip, alignment of deflection sensors) the good agreement of different calibration techniques, not only helps demonstrate their legitimacy, but it also reinforces the legitimacy of AFM as a quantitative tool for the study of nanotribology. ${ }^{32}$

The stress determination was made by measuring the curvature of the substrate by means of stylus profilometry and by applying Stoney's equation, as described in detail elsewhere. ${ }^{33}$ The hardness of the films was measured employing a NanoIndenter II from NanoInstruments with loads of $20 \mathrm{mN}$. The film hardness and elastic modulus were obtained according to the Oliver and Pharr method. ${ }^{34}$ The final hardness values were taken as the average of 6 indentations carried out in different spots for penetration depths of about $40 \mathrm{~nm}$ that are less than $20 \%$ of the samples thickness.

\section{RESULTS AND DISCUSSION}

The effects of the thermal annealing on the film composition can be followed in Fig. 1. In this figure, the atomic concentrations of hydrogen and fluorine, determined respectively by ERDA and RBS, are presented as a function of the temperature. As it is clear from the picture, both concentrations remain essentially constant for annealing temperatures up to $300{ }^{\circ} \mathrm{C}$, followed by a strong decrease for higher temperatures. Fluorine-loss was also determined by XPS. In this case, the same trend was observed, but the spectra taken from the near-surface layers showed complete fluorine depletion for annealing at $600{ }^{\circ} \mathrm{C}$. For comparison, in hard a-C:H films the hydrogen concentration remains unchanged for annealing up to $500{ }^{\circ} \mathrm{C} .{ }^{35}$

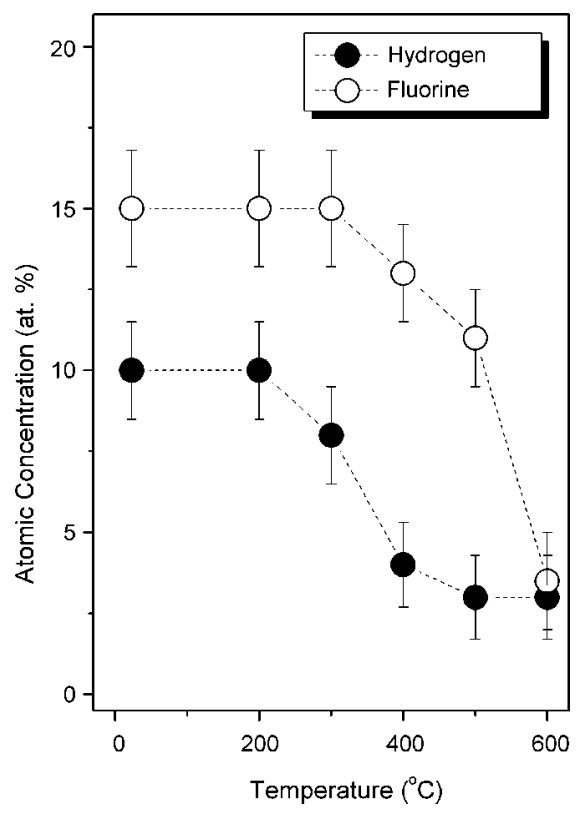

FIG. 1. Hydrogen and fluorine contents as functions of the annealing temperature. The lines are only to guide the eyes.

The atomic density as a function of the temperature is plotted in Fig. 2. In spite of large error bars, the results indicate a tendency of atomic density reduction up 33\%, drops over the range $25{ }^{\circ} \mathrm{C}$ and $600{ }^{\circ} \mathrm{C}$. These results show that the loss of hydrogen and fluorine determined by IBA is accompanied by mass loss, as already observed before by gas effusion experiments that reveal a strong effusion of $\mathrm{CF}_{4}$-related species starting at these temperatures. ${ }^{28}$ Moreover, it was determined by stylus profilometry that the variation of the film thickness was negligible even after $600{ }^{\circ} \mathrm{C}$

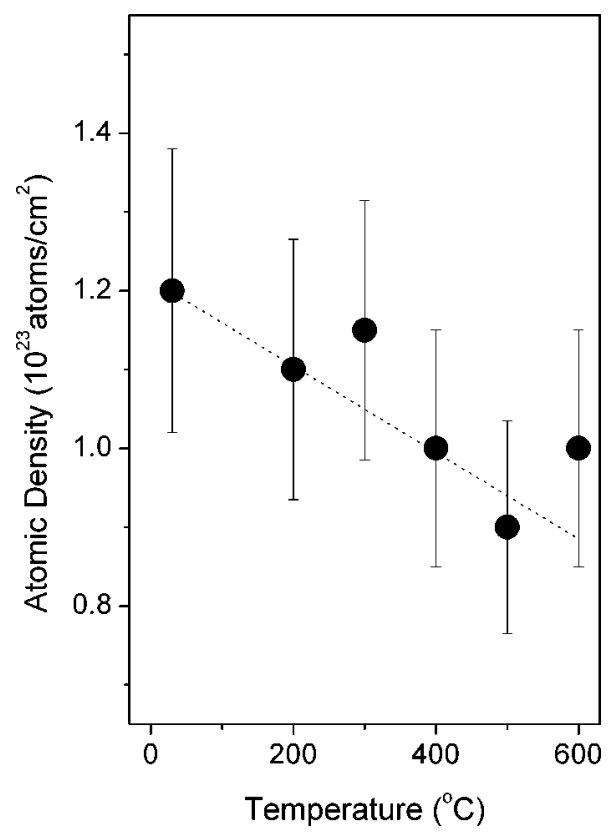

FIG. 2. Film atomic density as a function of the annealing temperatures. The line is only to guide the eyes. 


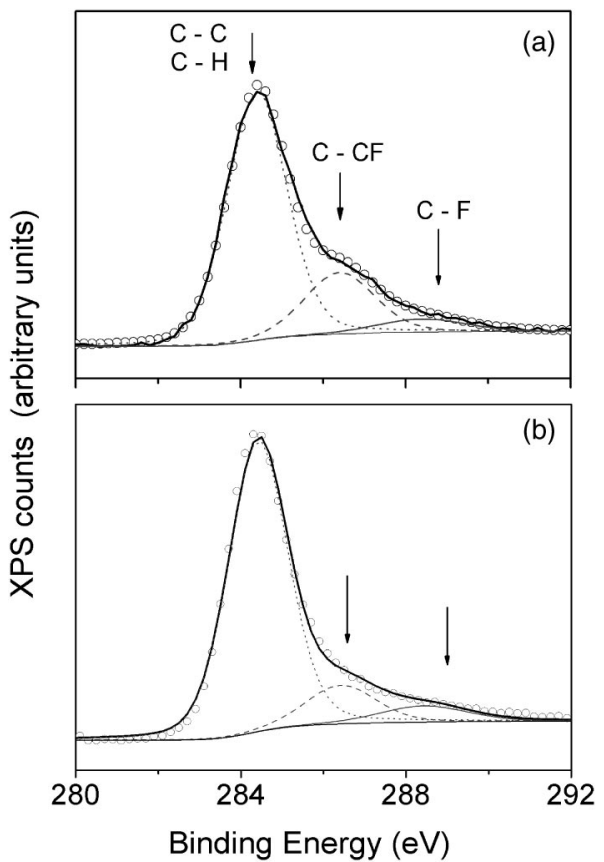

FIG. 3. XPS spectra obtained from a sample annealed at $200{ }^{\circ} \mathrm{C}$ (a) and at $500{ }^{\circ} \mathrm{C}$ (b). The figure also shows the simulated spectra by using Voigt peaks and the Shirley background. Arrows indicate the positions of the main features of these spectra.

annealing. On the other hand, relevant thickness changing was observed for annealed fluorine rich a-C:F films. ${ }^{13-16} \mathrm{In}$ these cases, the thickness reduction achieved $30 \%$ or more of the original film thickness. The film shrinkage indicates that they did not have highly cross-linked structures, as supported by the presence of $\mathrm{C}-\mathrm{F}_{3}$ and $\mathrm{C}-\mathrm{F}_{2}$ groups by both infrared and XPS results. ${ }^{13,36}$

The chemical state of the carbon atoms at the surface was probed by XPS. In Fig. 3 two spectra are shown. The upper spectrum was obtained from a film annealed at $200{ }^{\circ} \mathrm{C}$. It is identical of a spectrum taken from an as-deposited a-C:F film (not shown). The carbon peak is dominated by the $\mathrm{C}-\mathrm{C} / \mathrm{C}-\mathrm{H}$ bonds at $285.1 \mathrm{eV}$ with a shoulder at the higher binding energy side. The band at $289.5 \mathrm{eV}(\mathrm{C}-\mathrm{F})$ corresponds to the $\mathrm{C}_{1 s}$-binding energy of a carbon atom directed bonded to a fluorine atom, while the band at $287 \mathrm{eV}(\mathrm{C}-\mathrm{CF})$ is related to carbon atoms bonded to another carbon in the neighborhood of a fluorine atom. ${ }^{37}$ The intensity of the $\mathrm{C}-\mathrm{F}_{2}$ and $\mathrm{C}-\mathrm{F}_{3}$ bands are negligible, confirming the high degree of structural interconnectivity of the films. The bottom spectrum is from a sample annealed at $500{ }^{\circ} \mathrm{C}$, One can observe that the increase of the annealing temperature does not induce any noticeable changes in the carbon chemical environment despite the lower fluorine content. Finally, the spectrum taken from the sample annealed at $600{ }^{\circ} \mathrm{C}$ presents one single peak at $285.1 \mathrm{eV}$ and it confirms the formation of a fluorine depleted layer at the film surface after annealing at this temperature. All samples have a small oxygen surface contaminant. The position of the $\mathrm{C}-\mathrm{O}$ peak is $285.8 \mathrm{eV}$. However, since the spectrum obtained from the samples treated at higher temperatures shows that the $\mathrm{C}-\mathrm{O}$ peak in-



FIG. 4. Raman spectra obtained from the as-deposited and annealed samples.

tensity is negligible, we assumed that the oxygen contamination is not important for the interpretation of the XPS data.

Raman results are presented in Fig. 4. The Raman spectra consist of two intense and partially overlapping bands observed in the spectral region between 1100 and $1700 \mathrm{~cm}^{-1}$, the $G$ - and $D$-bands. The first one corresponds to the $G$-line associated to the optically allowed $E_{2 g}$-zone center of crystalline graphite and the second one corresponds to the disorder-allowed zone edge modes of graphite that became Raman active due to the lack of long-range order in amorphous carbon-based materials. ${ }^{38}$ The spectrum from the asdeposited sample is similar to the one obtained from films annealed at low temperatures (up to $300{ }^{\circ} \mathrm{C}$ ) and resembles the spectra typical of Raman spectra obtained from diamondlike films. In fact, Raman spectra obtained from polymeric films have a strong luminescence background that is absent in all spectra presented in Fig. $4 .^{22,29}$ It is clear from the spectra, that a progressive increase of the relative intensity of the $D$-band for higher annealing temperatures occur. Both the peak position and the bandwidth of the $G$-band as well as the $I_{D} / I_{G}$ intensity ratio are sometimes used, as graphitization indices. ${ }^{39}$ With this regard, the line narrowing, especially the narrowing of the $G$-band, undoubtedly reflects an increase of structural order of the graphitic component.

The Raman spectra were deconvoluted with a computer code that takes into account the luminescence background, by using a second-order polynomial, and fits the Raman bands with Gaussian lines. ${ }^{40}$ The position, width and intensity of each line were obtained by a least-square fitting algorithm and the values for the $G$-bands are presented as a function of the temperature in Fig. 5. While the position $\left(\sim 1370 \mathrm{~cm}^{-1}\right)$ and width $\left(\sim 300 \mathrm{~cm}^{-1}\right)$ of the $D$-band are nearly independent on the annealing temperature, the posi- 



FIG. 5. (a) $G$-band width; (b) $G$-band position, and (c) $I_{D} / I_{G}$ as functions of the annealing temperatures.

tion of the $G$-band $\left(\omega_{G}\right)$ shifts from 1555 to $1581 \mathrm{~cm}^{-1}$ and the $I_{D} / I_{G}$ intensity ratio increases with the temperature, the width of the $G$-band $\left(\Gamma_{G}\right)$ reduces from 141 to $101 \mathrm{~cm}^{-1}$. For temperatures equal or higher than $500{ }^{\circ} \mathrm{C}$ all the Raman parameters are nearly constant. These results are usually interpreted as being due to an increase in the size and/or the number of the graphitic domains. ${ }^{39,40}$ We can obtain a transition temperature from the amorphous diamond like structure to the more graphitic-like film following the same criteria adopted previously by Müller and collaborators, i.e., the turning-points of the $I_{D} / I_{G}, \Gamma_{G}$, and $\omega_{G}$ curves as functions of the temperature. ${ }^{26}$ In this way, the transition temperature is around $350{ }^{\circ} \mathrm{C}$, in good agreement with previous results for a-C:F films deposited by PECVD with similar fluorine content. ${ }^{26}$ Raman results obtained from carbon films annealed in vacuum in the same temperature range (200 and $700{ }^{\circ} \mathrm{C}$ for $30 \mathrm{~min}$ ) present similar trend. In fact, the transition temperature for annealed hard sputtered a-C films is around $350{ }^{\circ} \mathrm{C},{ }^{41}$ while for nitrogenated a-C: $\mathrm{H}$ films is of the order of $400{ }^{\circ} \mathrm{C}$ and $500{ }^{\circ} \mathrm{C}$ for a-C:H films. ${ }^{26,35}$

The contact angle was measured for as-deposited and annealed samples and the obtained results are presented in Fig. 6 . We observe a slight increase of the contact angle for temperatures higher than $300{ }^{\circ} \mathrm{C}$. The obtained contact angles are in the same range normally reported in the literature for graphite at room-temperature, $80^{\circ}-90^{\circ} .{ }^{42}$ As it was observed by XPS, the surface of the film annealed at $600{ }^{\circ} \mathrm{C}$ is free from fluorine and a reduction of the surface wettability for the higher annealing temperatures should be expected since it is known that the fluorine surface concentration controls the surface energy. ${ }^{43}$ However, the Raman results suggest the formation of larger graphitic domains for annealing temperatures higher than $300{ }^{\circ} \mathrm{C}$, and so, a more hydrophobic sur-

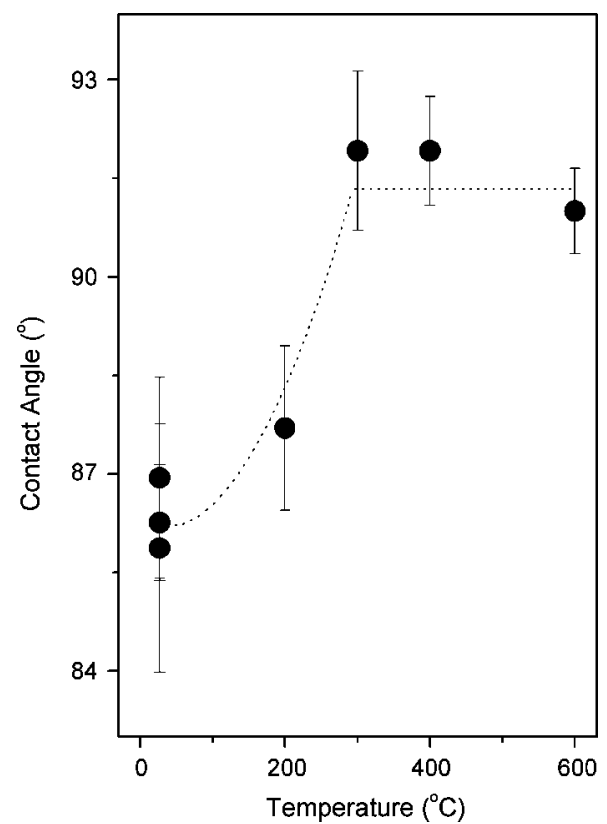

FIG. 6. Contact angle as a function of the annealing temperature. The line is only to guide the eyes.

face. In this way, the measured contact angles reflect the competition between these two opposite trends and only a slight increase was observed.

AFM was employed to investigate the topographical changes induced by the annealings. In Fig. 7 we show the
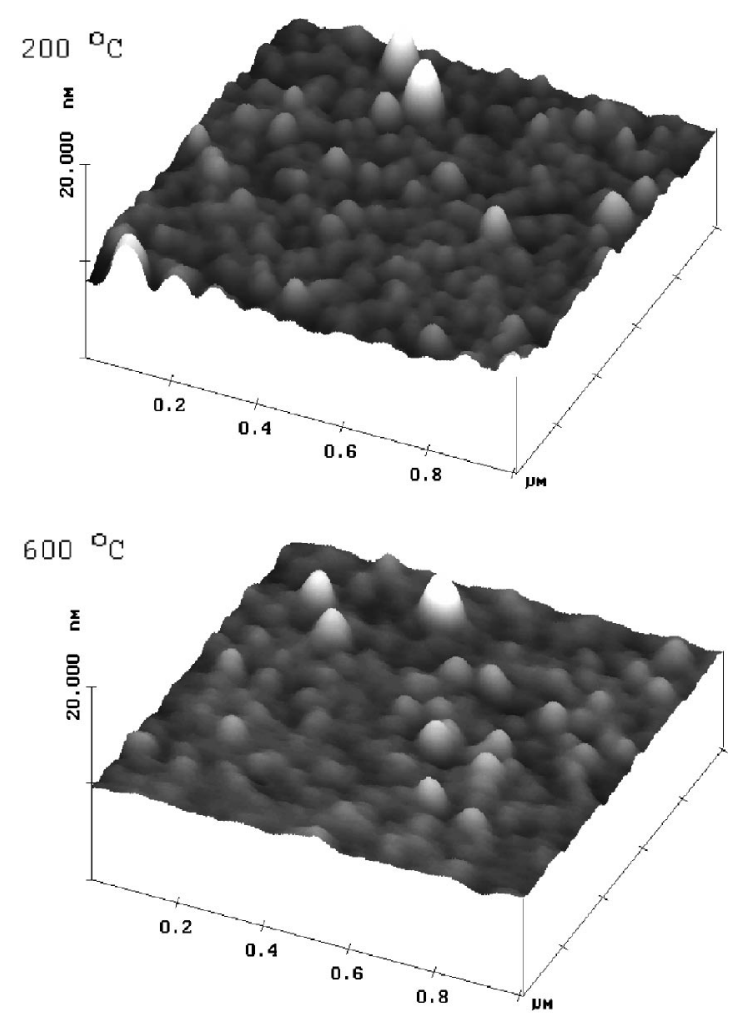

FIG. 7. AFM images obtained from a-C:F films annealed at two different temperatures, as indicated. 


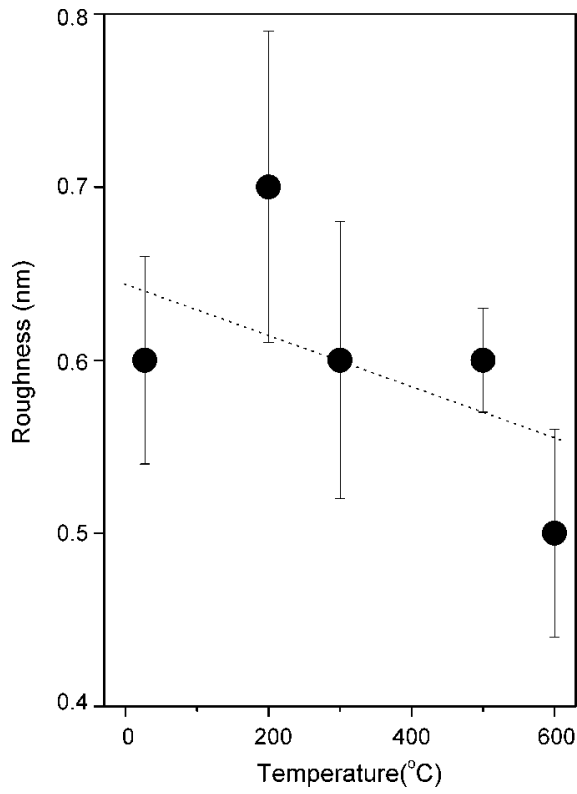

FIG. 8. RMS roughness as a function of the annealing temperature.

AFM images obtained from a sample annealed at $200{ }^{\circ} \mathrm{C}$ and from a sample annealed at $600{ }^{\circ} \mathrm{C}$. A slight decrease of the roughness with the increasing annealing temperatures can be seen in Fig. 8. It occurs despite the fact that the Raman results indicate a progressive graphitization of the films upon annealing. In effect, there are reports in the literature that show a direct correlation between the fraction of $s p^{2}$ carbonbonds and the surface roughness. ${ }^{44}$ However, we cannot only ascribe the roughness to the number and size of the graphitic domains. Other surface modifications, as chemical composition changes observed by XPS (fluorine depletion), can play an important role.

The adhesion forces and the friction coefficients are plotted as functions of the annealing temperature in Fig. 9. The friction coefficients were easily obtained from the lateral forces versus load forces curve. In fact, a linear behavior was observed, also indicating a multi-asperity contact regime. It is clear from Figs. 6 and 9, that the friction coefficients and the contact angle have an opposite behavior, while the adhesion forces and friction coefficients have the same trend. Recently, a correlation between friction forces measured by AFM and the contact angles of a-C:F films with different fluorine content was shown, with the friction coefficient decreasing with the increase of the contact angle. ${ }^{5}$ It was suggested that the sliding friction forces at nanometer scale are determined by two competitive processes. ${ }^{45}$ Depending on the surface wettability, one of the processes dominates. The first process is the thermally activated stick and slip behavior when friction is originated in the adhesive forces between the two surfaces in contact. Moreover, capillary effects due to water vapor condensation must be taken into consideration, especially for measurements performed in air. ${ }^{46}$ In this case, the friction results can be interpreted as being due to the kinetics of nucleation of water meniscus between the moving parts: the higher the surface hydrophobicity, the less impor-

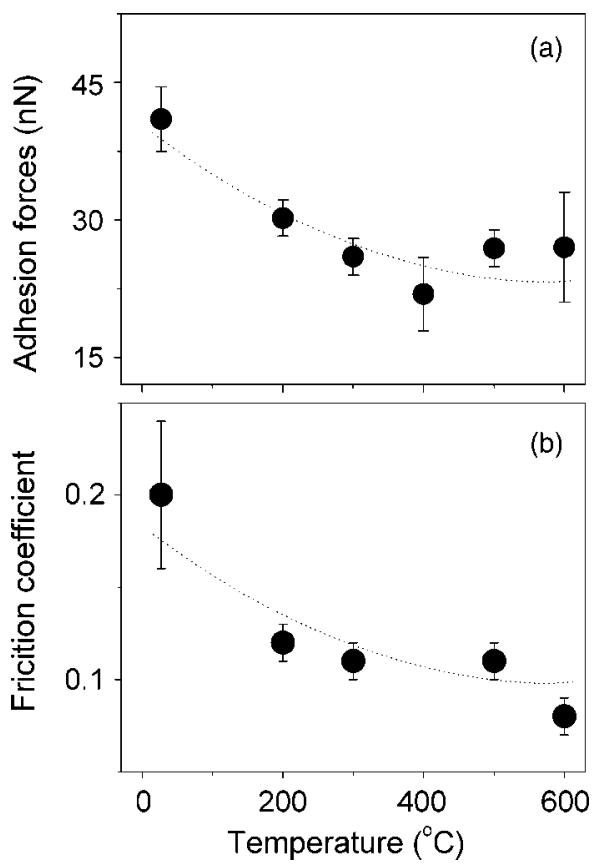

FIG. 9. (a) Adhesion forces and (b) friction coefficient as functions of the annealing temperature.

tant is the water vapor condensation for the nanoscale friction process. ${ }^{5,45}$ The present results show a surface wettability reduction and a decrease of the friction coefficient, confirming the influence of the capillary condensation on the nanoscale friction.

The mechanical properties are presented in Figs. 10 and 11. The hardness and the compressive internal stress are constant up to $300{ }^{\circ} \mathrm{C}$ and then decrease following the same trend observed for the atomic density of the films. It is well

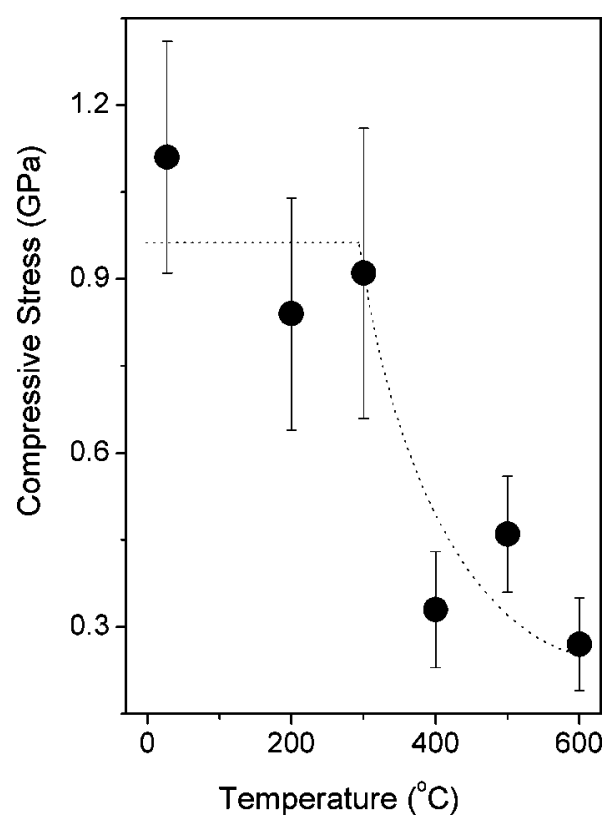

FIG. 10. Compressive internal stress as a function of the temperature. The line is only to guide the eyes. 


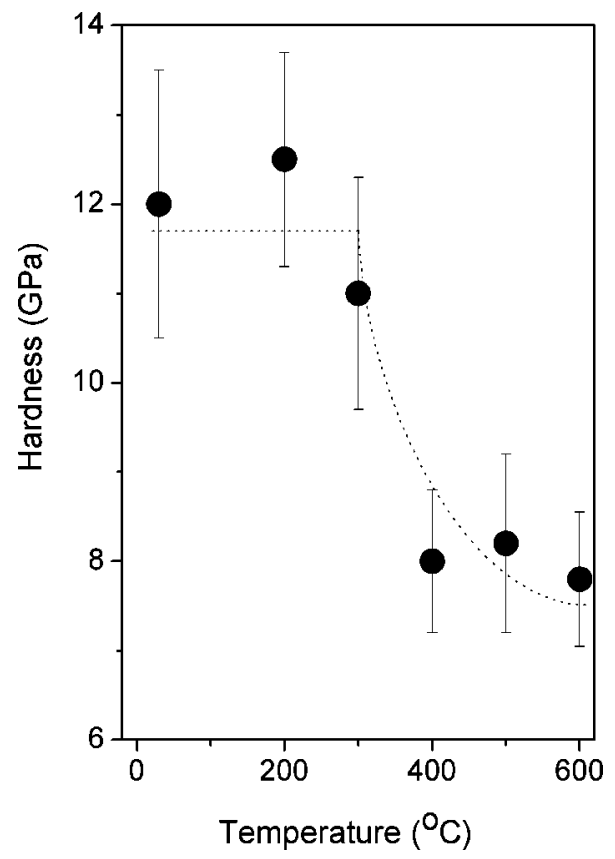

FIG. 11. Film hardness a function of the temperature. The line is only to guide the eyes.

known that hard carbon films are amorphous cross-linked structures, whose degree of cross-link and carbon hybridization depends on the ion bombardment during film growth. ${ }^{1}$ The mass losses upon thermal treatment, together with the increase of the fraction of $s p^{2}$ hybridized carbon atoms, result in amorphous structures with a lower degree of crosslinking, explaining the observed deterioration of the mechanical properties of the a-C:F films.

\section{SUMMARY AND CONCLUSIONS}

For low dielectric constant a-C:F films, submitted to annealing treatment, the critical temperature for the stability of the composition and dielectric properties is around $300{ }^{\circ} \mathrm{C}$, with the observation of mass loss and film shrinkage occur, together with an increase of the dielectric constant. ${ }^{13,19}$ In this work, we submitted hard a-C:F films deposited by PECVD to isochronal annealing in the temperature range of 200 to $600{ }^{\circ} \mathrm{C}$. These films, with much lower fluorine content than the former ones, have a dense cross-linked structure characterized by the absence of $\mathrm{C}-\mathrm{F}_{3}$ and $\mathrm{C}-\mathrm{F}_{2}$ bonds as revealed by XPS and infrared spectroscopy. ${ }^{2,22,29}$ However, we found that despite the higher structural interconnectivity and negligible thickness changing significant material loss occurs as a result of the annealing. We verified that at temperatures higher than $300{ }^{\circ} \mathrm{C}$ both hydrogen and fluorine losses occur while the film thickness changes only a few per cent, resulting in an important atomic density reduction. The mass loss was accompanied by structural modifications, with Raman results suggesting a progressive graphitization of the film and a transition temperature from an amorphous diamond-like film structure to a nanocrystalline graphitic material around $350{ }^{\circ} \mathrm{C}$. The increase of the fraction of carbon atoms with $s p^{2}$ hybridization, together with the mass loss, can explain the lower degree of cross-linking, and consequently the low atomic density observed for films annealed at temperatures higher than $300{ }^{\circ} \mathrm{C}$. This structural modification also explains the modifications observed in the mechanical properties. In fact, a direct correlation between the reduction of the film hardness and internal stress and the atomic density can be done.

The thermal treatment also induces modifications on the surface hydrophobicity and friction behavior of the films. The treated films are slightly more hydrophobic than the asdeposited one, while the friction coefficient decreases upon the annealing temperatures. The observed correlation between the contact angle and the friction coefficient reinforced the importance of the capillary condensation kinetics in the friction process that occurs at nanometer scale.

\section{ACKNOWLEDGMENTS}

This work was partially supported by the Brazilian Agencies: Conselho Nacional de Desenvolvimento Científico e Tecnológico (CNPq), Coordenação de Aperfeiçoamento de Pessoal de Nível Superior (CAPES) and Fundação de Amparo à Pesquisa do Estado do Rio de Janeiro (FAPERJ). The Authors would like to thank Professor A. R. Zanatta for the Raman measurements and Professor R. Prioli for the help with the AFM experiments.

${ }^{1}$ J. Robertson, Prog. Solid State Chem. 21, 199 (1991).

${ }^{2}$ L. G. Jacobsohn, D. F. Franceschini, M. E. H. Maia da Costa, and F. L. Freire, Jr., J. Vac. Sci. Technol. A 18, 2230 (2000).

${ }^{3}$ R. E. Sah, B. Dischler, A. Bubenzer, and P. Koidl, Appl. Phys. Lett. 46, 739 (1985).

${ }^{4}$ A. Grill and V. Patel, Diamond Films Technol. 6, 13 (1996).

${ }^{5}$ R. Prioli, L. G. Jacobsohn, M. E. H. Maia da Costa, and F. L. Freire, Jr., Tribol. Lett. 15, 177 (2003).

${ }^{6}$ M. Grischke, K. Benilogua, K. Trojan, and H. Dimigen, Surf. Coat. Technol. 74-75, 739 (1995).

${ }^{7}$ C. Donnet, J. Fontaine, A. Grill, V. Patel, C. Janhes, and M. Belin, Surf. Coat. Technol. 94-95, 531 (1997).

${ }^{8}$ R. S. Butter, D. R. Waterman, A. H. Lettington, R. T. Ramos, and E. J. Fordham, Thin Solid Films 311, 107 (1997).

${ }^{9}$ M. Hakovirta, D. H. Lee, X. M. He, and M. Nastasi, J. Vac. Sci. Technol. A 19, 782 (2001).

${ }^{10}$ R. Gilmore and R. Hauert, Thin Solid Films 398-399, 199 (2001).

${ }^{11}$ H. Ji, A. Côté, D. Koshel, B. Terreault, G. Abel, P. Ducharme, G. Ross, S. Savoie, and M. Gagné, Thin Solid Films 405, 104 (2002).

${ }^{12}$ J. A. Theil, J. Vac. Sci. Technol. B 17, 2397 (1999).

${ }^{13}$ K. Endo and T. Tatsumi, J. Appl. Phys. 78, 1370 (1995).

${ }^{14}$ K. Endo and T. Tatsumi, Appl. Phys. Lett. 68, 2864 (1996).

${ }^{15}$ A. Grill, V. Patel, and C. Jahnes, J. Electrochem. Soc. 145, 1649 (1998).

${ }^{16}$ H. Yokomichi, T. Hayashi, and A. Masuda, Appl. Phys. Lett. 72, 2704 (1998).

${ }^{17}$ J. P. Chang, H. W. Krautter, W. Zhu, R. L. Opila, and C. S. Pai, J. Vac. Sci. Technol. A 17, 2969 (1999).

${ }^{18}$ N. Biswas, H. R. Harris, X. Wang, G. Celebi, H. Temkin, and S. Gangopadhyay, J. Appl. Phys. 89, 4417 (2001).

${ }^{19}$ A. Grill, Diamond Relat. Mater. 10, 234 (2001)

${ }^{20}$ J. M. Shieh, K. C. Tsai, S. C. Suen, and B. T. Dai, J. Vac. Sci. Technol. B 20, 1388 (2002).

${ }^{21}$ K. Takahashi, T. Mitamura, K. Ono, Y. Setsuhara, A. Itoh, and K. Tachibana, Appl. Phys. Lett. 82, 2476 (2003).

${ }^{22}$ R. d'Agostino, R. Lamendola, P. Favia, and A Giquel, J. Vac. Sci. Technol. A 12, 308 (1994).

${ }^{23}$ F. L. Freire, Jr., J. Non-Cryst. Solids 304, 251 (2002).

${ }^{24}$ L. Valentini, M. C. Bellachioma, S. I. -U. Ahmed, G. Bregliozzi, Y. Gerbi, H. Haefke, L. Lozzi, S. Santucci, and J. M. Kenny, J. Vac. Sci. Technol. 
A 21, 1964 (2003).

${ }^{25}$ Y. Ma, H. Yang, J. Guo, C. Sathe, A. Agui, and J. Nordgren, Appl. Phys. Lett. 72, 3353 (1998).

${ }^{26}$ U. Müller, R. Hauert, B. Oral, and M. Tobler, Surf. Coat. Technol. 76-77, 367 (1995).

${ }^{27}$ H. Yong, D. J. Tweet, Y. Ma, and T. Nguyen, Appl. Phys. Lett. 73, 1514 (1998).

${ }^{28}$ L. G. Jacobsohn, S. S. Camargo, Jr., and F. L. Freire, Jr., Diamond Relat. Mater. 11, 1831 (2002)

${ }^{29}$ L. G. Jacobsohn, M. E. H. Maia da Costa, V. J. Trava-Airoldi, and F. L. Freire, Jr., Diamond Relat. Mater. 12, 2037 (2003).

${ }^{30}$ J. M. Neumeister and W. A. Ducker, Rev. Sci. Instrum. 65, 2527 (1994).

${ }^{31}$ E. Liu, B. Blanpain, J. P. Celis, and J. R. Roos, J. Appl. Phys. 84, 4859 (1998).

${ }^{32}$ R. G. Cain, M. G. Reitsma, S. Biggs, and N. W. Page, Rev. Sci. Instrum. 723304 (2001).

${ }^{33}$ M. M. Lacerda, F. L. Freire, Jr., R. Prioli, C. M. Lepinski, and G. Mariotto, J. Vac. Sci. Technol. A 17, 2811 (1999).

${ }^{34}$ W. C. Oliver and G. M. Pharr, J. Mater. Res. 7, 1564 (1992).

${ }^{35}$ F. L. Freire, Jr., C. A. Achete, G. Mariotto, and R. Canteri, J. Vac. Sci. Technol. A 12, 3048 (1994).
${ }^{36}$ L. Valentini, E. Braca, J. M. Kenny, G. Fedosenko, J. Engemann, L. Lozzi, and S. Santucci, Diamond Relat. Mater. 11, 1100 (2002).

${ }^{37}$ S. Agraharam, D. H. Hess, P. A. Kohl, and S. Bistrup Allen, J. Vac. Sci. Technol. A 17, 3265 (1999).

${ }^{38}$ F. Tuinstra and J. L. Koenig, J. Chem. Phys. 53, 1126 (1970).

${ }^{39}$ R. O. Dillon, J. A. Woollam, and V. Katkanant, Phys. Rev. B 29, 3482 (1984).

${ }^{40}$ G. Mariotto, F. L. Freire, Jr., and C. A. Achete, Thin Solid Films 241, 255 (1994).

${ }^{41}$ L. G. Jacobsohn, R. Prioli, F. L. Freire, Jr., G. Mariotto, M. M. Lacerda, and Y. W. Chung, Diamond Relat. Mater. 9, 680 (2000).

${ }^{42}$ A. W. Adamson and A. P. Gast, Physical Chemistry of Surfaces, 6th ed. (Wiley, New York, 1997).

${ }^{43}$ R. D. van de Grampel, W. Ming, A. Gildenpfennig, J. Laven, H. H. Brongersma, G. de With, and R van der Linde, Langmuir 20, 145 (2004).

${ }^{44}$ Y. Lifshitz, G. D. Lempert, and E. Grossman, Phys. Rev. Lett. 72, 2753 (1994).

${ }^{45}$ E. Riedo, F. Lévy, and H. Brune, Phys. Rev. Lett. 88, 185505 (2002).

${ }^{46}$ A. Opitz, S. I.-U. Ahmed, J. A. Schaefer, and M. Scherge, Surf. Sci. 504, 199 (2002). 